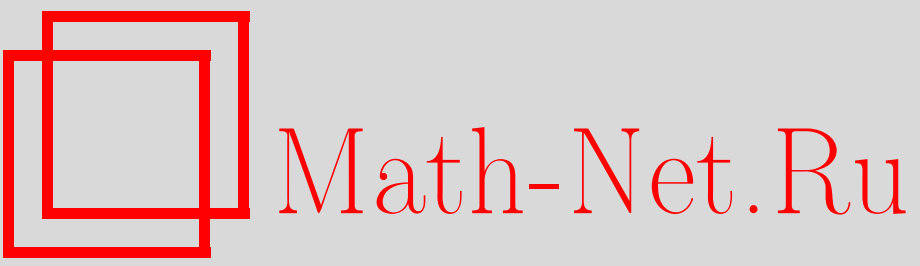

А. А. Переломова, С. Б. Лебле, Взаимодействие вихревых и акустических волн. От общих уравнений к интегрируемым, ТМФ, 2005, том 144, номер 1, 171-181

DOI: https://doi.org/10.4213/tmf1843

Использование Общероссийского математического портала Math-Net.Ru подразумевает, что вы прочитали и согласны с пользовательским соглашением

http://www.mathnet.ru/rus/agreement

Параметры загрузки:

IP: 3.85 .5 .30

26 апреля 2023 г., 14:43:02 
ТЕОРЕТИЧЕСКАЯ

И МАТЕМАТИЧЕСКАЯ

ФИЗИКА

Том 144, № 1

июль, 2005

(C) 2005 г.

А. А. Переломова*, С.Б. Лебле*

\title{
ВЗАИМОДЕЙСТВИЕ ВИХРЕВЫХ И АКУСТИЧЕСКИХ ВОЛН. ОТ ОБЩИХ УРАВНЕНИЙ К ИНТЕГРИРУЕМЫМ
}

\begin{abstract}
Уравнения для $(2+1)$-мерного возмущения в пограничном слое разложены на собственные моды: вихревую волну и две акустических волны. Уравнения состояния (аппроксимация рядом Тейлора) предполагаются произвольными. Моды определяются посредством локальных уравнений связи, которые выделяются из обшей системы, линеаризованной на потоке в пограничном слое. Каждая такая связь определяет инвариантное подпространство и соответствующий проектор. Нелинейное уравнение для вихревой волны исследуется с помощью специальной ортогональной системы координат, основанной на линиях тока. Преобразования Лапласа и Мутара связывают уравнения для ортогональных кривых с уравнениями Лапласа. Нелинейность определяет правильный вид взаимодействия между вихревым и акустическими полями возмущений в пограничном слое, которые определяются как результат проектирования на подпространство решений уравнения Орра-Зоммерфельда для волны Толлмина-Шлихтинга (линейной вихревой волны) и при помощи соответствующей процедуры для акустических волн. Предложен новый механизм нелинейного резонансного управления волной Толлмина-Шлихтинга с помощью звуковых волн посредством четырехволнового взаимодействия.
\end{abstract}

Ключевые слова: механика жидкостей и газов, пограничный слой, проекторы на собственные моды, волны Толлмина-Шлихтинга, преобразование Лапласа, преобразование Мутара, акустика, $N$-волновые системы.

\section{1. ВВЕДЕНИЕ. ОСНОВНЫЕ УРАВНЕНИЯ}

В настоящей работе используются две основные идеи, причем обе связаны с надлежашим выбором переменных. Первая идея - ввести новые зависимые переменные в такой комбинации (с операторными коэффициентами), чтобы зафикисировать соотношения, определяюшие конкретную ветвь (корень) дисперсионного уравнения основной системы, линеаризованной на основном потоке [1]. Вторая идея - так скомбинировать независимые переменные, чтобы введенные на первом шаге преобразования зависимые

* Technical University of Gdańsk, ul. G. Naturowicza, 11/12, 80-952, Gdańsk-Wrzeszcz, Poland. E-mail: anpe@mif.pg.gda.pl, leble@mif.pg.gda.pl 
переменные остались неизменными. Эта процедура сопровождается введением ортогональных координатных линий. Такой подход представляет собой попытку ограничиться минимальным числом переменных для выбранного типа движения (моды), избегая введения граничных подслоев.

Первая цель достигается с помощью ортогональных матрично-дифференциальных операторов проектирования, чьи структурные особенности определяют упомянутые соотношения, связываюшие компоненты вектора состояния рассматриваемой системы. Явный вид таких операторов дан в п. 2.3. Вторая цель до некоторой степени аналогична той, которую преследует метод годографа: в качестве координат выбирается одна из новых зависимых переменных вместе с сопутствующей ей (локально) ортогональной переменной. Эта задача решается с помошью объединенного преобразования Лапласа-Мутара.

Пример гидротермодинамики был выбран нами потому, что в нем проявляются характерные трудности, связанные с неединственностью моды поля в целом, неоднородностью среды и нелинейностью. Описание возмушений и их взаимодействия восходит к работе [2] и интенсивно изучалось в работе [3]. История вопроса изложена в работе [4]. Теория акустических солитонов и сопутствующих возмущений в неоднородных/слоистых средах рассматривается в работе [5].

Начнем с введения следующих обозначений: $\rho, p$-возмущения плотности и давления, $e, T$ - удельной внутренней энергии (на единицу массы) и температуры, $\eta, \varsigma-$ сдвиговая и объемная вязкости, $\chi$ - коэффициент теплопроводности, $\vec{v}$ - вектор скорости.

Далее запишем уравнения состояния в виде рядов Тейлора для энергии (калорическое):

$$
\rho_{0} e=E_{1} p+\frac{E_{2} p_{0}}{\rho_{0}} \rho+\frac{E_{3}}{p_{0}} p^{2}+\frac{E_{4} p_{0}}{\rho_{0}^{2}} \rho^{2}+\frac{E_{5}}{\rho_{0}} p \rho+\cdots,
$$

и для температуры (термическое):

$$
T=\frac{\Theta_{1}}{\rho_{0} C_{v}} p+\frac{\Theta_{2} p_{0}}{\rho_{0}^{2} C_{v}} \rho+\cdots .
$$

Фоновые значения отмечены индексом "нуль", $C_{v}$ - удельная теплоемкость (на единицу массы) при постоянном объеме, $E_{i}, \Theta_{i}$ - безразмерные коэффициенты.

Рассмотрим двумерньй поток в координатах $x$ (в направлении течения от переднего фронта модели), $z$ (по нормали к координате поверхности модели) и

$$
\vec{v}=(u, w)+\vec{u}_{0},
$$

где $\vec{u}_{0}=\left(U_{0}(z), 0\right)$ - постоянная составляющая скорости в направлении потока, а $(u, w)=\vec{v}^{\prime}-$ флуктуации скорости.

С учетом уравнений (1)-(3) основная система имеет вид

$$
\begin{aligned}
\frac{\partial p}{\partial t}+V_{0} \frac{\partial p}{\partial x}+\epsilon^{-2}\left(\frac{\partial u}{\partial x}+\frac{\partial w}{\partial z}\right) & =\tilde{\varphi}_{1}, \\
\frac{\partial u}{\partial t}+V_{0} \frac{\partial u}{\partial x}+\phi w+\frac{\partial p}{\partial x}-\operatorname{Re}^{-1} \Delta u-R^{-1}\left(\frac{\partial^{2} u}{\partial x^{2}}+\frac{\partial^{2} w}{\partial x \partial z}\right) & =\tilde{\varphi}_{2}, \\
\frac{\partial w}{\partial t}+V_{0} \frac{\partial w}{\partial x}+\frac{\partial p}{\partial z}-\operatorname{Re}^{-1} \Delta w-R^{-1}\left(\frac{\partial^{2} u}{\partial x \partial z}+\frac{\partial^{2} w}{\partial z^{2}}\right) & =\tilde{\varphi}_{3},
\end{aligned}
$$


где $\epsilon=U_{\infty} / c$-число Маха, $\operatorname{Re}=U_{\infty} l_{0} \rho_{0} / \eta$-число Рейнольдса, $R=U_{\infty} l_{0} \rho_{0} /(\eta / 3+\varsigma)$. Вводя для правой части векторный нелинейный член

$$
\widetilde{\varphi}=\left(\begin{array}{c}
-u \frac{\partial p}{\partial x}-w \frac{\partial p}{\partial z}+\left(\frac{\partial u}{\partial x}+\frac{\partial w}{\partial z}\right)\left[Z p+\epsilon^{-2} S \rho\right] \\
-u \frac{\partial u}{\partial x}-w \frac{\partial u}{\partial z}+\rho \frac{\partial p}{\partial x} \\
-u \frac{\partial w}{\partial x}-w \frac{\partial w}{\partial z}+\rho \frac{\partial p}{\partial z}
\end{array}\right),
$$

можно переписать систему (4) в виде

$$
\frac{\partial}{\partial t} \varphi+L \varphi=\tilde{\varphi}, \quad \varphi=\left(\begin{array}{c}
p \\
u \\
w
\end{array}\right)
$$

где $\varphi$ - вектор состояния жидкости (сплошной среды) и $L$ - матричньй оператор, элементы которого находятся из системы (4). Постоянные $Z$ и $S$ определены следующим образом:

$$
Z=\frac{1}{E_{1}}\left(-1+2 \frac{1-E_{2}}{E_{1}} E_{3}+E_{5}\right), \quad S=\frac{1}{1-E_{2}}\left(1+E_{2}+2 E_{4}+\frac{1-E_{2}}{E_{1}} E_{5}\right) .
$$

Постоянная $c=\sqrt{p_{0}\left(1-E_{2}\right) /\left(\rho_{0} E_{1}\right)}-$ линейная скорость звука при $U_{0}=0$. Данное матричное представление позволяет осушествить дальнейшую процедуру проектирования и делает ее более прозрачной.

\section{2. МОДЫ В ЛИНЕЙНОМ ПРИБЛИЖЕНИИ}

Результаты данного раздела были кратко представлены в работе [6].

Используем линейный вариант системы (6) в качестве основы для определения моделей и следуюшие обозначения для безразмерных переменных:

$$
\begin{gathered}
V_{0}(z)=\frac{U_{0}(z)}{U_{\infty}}, \quad \phi(z)=V_{0 z}(z) l_{0}, \\
x_{*}=\frac{x}{l_{0}}, \quad u_{*}=\frac{u}{U_{\infty}}, \quad t_{*}=\frac{t U_{\infty}}{l_{0}}, \quad p_{*}=\frac{p}{\rho_{0} U_{\infty}^{2}} .
\end{gathered}
$$

Величина $U_{\infty}$ - значение скорости потока вдали от гранищы, а $l_{0}$ - ширина пограничного слоя. В новых переменных (звездочки опушены) линейный аналог (6) имеет вид

$$
\begin{aligned}
\frac{\partial p}{\partial t}+V_{0} \frac{\partial p}{\partial x}+\epsilon^{-2}\left(\frac{\partial u}{\partial x}+\frac{\partial w}{\partial z}\right) & =0, \\
\frac{\partial u}{\partial t}+V_{0} \frac{\partial u}{\partial x}+\phi w+\frac{\partial p}{\partial x}-\mathrm{Re}^{-1} \Delta u-R^{-1}\left(\frac{\partial^{2} u}{\partial x^{2}}+\frac{\partial^{2} w}{\partial x \partial z}\right) & =0, \\
\frac{\partial w}{\partial t}+V_{0} \frac{\partial w}{\partial x}+\frac{\partial p}{\partial z}-\mathrm{Re}^{-1} \Delta w-R^{-1}\left(\frac{\partial^{2} u}{\partial x \partial z}+\frac{\partial^{2} w}{\partial z^{2}}\right) & =0 .
\end{aligned}
$$


2.1. Вихревая мода (Толлмина-Шлихтинга). Для введения вихревой моды обычно используется предел несжимаемой жидкости $(\epsilon=0)$ :

$$
\frac{\partial u}{\partial x}+\frac{\partial w}{\partial z}=0
$$

Другая связь полей скорости и давления получается из уравнений $(10),(11)$ и является, наверное, новой:

$$
2 c \phi \frac{\partial w}{\partial x}+\Delta p=0 .
$$

Традиционное для теории пограничного слоя $x$-однородное приближение позволяет использовать $k$-представление для операторов $\partial / \partial x \rightarrow-i k$. Уравнения (12) и (13) совместно определяют вектор моды Толлмина-Шлихтинга (ТШ):

$$
T=\left(\begin{array}{c}
p_{\mathrm{TS}} \\
u_{\mathrm{TS}} \\
w_{\mathrm{TS}}
\end{array}\right)=\left(\begin{array}{c}
1 \\
-\frac{1}{2 k^{2}} \frac{\partial}{\partial z} \frac{1}{\phi} \Delta \\
\frac{1}{2 i k \phi} \Delta
\end{array}\right) p_{\mathrm{TS}} .
$$

Уравнения (9)-(11) дают хорошо известное уравнение для ТШ-моды [4] в терминах функции тока $\Psi$ :

$$
\begin{gathered}
u=\frac{\partial \Psi}{\partial z}, \quad w=-\frac{\partial \Psi}{\partial x} \\
\Delta \frac{\partial \Psi}{\partial t}+V_{0} \Delta \frac{\partial \Psi}{\partial x}-\frac{\partial \Psi}{\partial x} \frac{\partial \phi}{\partial z}-\operatorname{Re}^{-1} \Delta^{2} \Psi=0 .
\end{gathered}
$$

Уравнение Орра-Зоммерфельда (ОЗ) следует из уравнения (15), когда $\Psi=\psi(z) \times$ $\exp (i k c t-i k x)$ :

$$
\left[V_{0}(z)-c\right]\left[\frac{\partial^{2} \psi}{\partial z^{2}}-k^{2} \psi\right]-\psi \frac{\partial \phi}{\partial z}=\frac{i}{\operatorname{Re} k}\left[\frac{\partial^{4} \psi}{\partial z^{4}}-2 k^{2} \frac{\partial^{2} \psi}{\partial z^{2}}+k^{4} \psi\right] .
$$

Это уравнение составляет основу теории устойчивости и для любой пары $(k, \mathrm{Re})$ определяет собственную функцию $\psi(z)$ и комплексную фазовую скорость $c=\omega / k=c_{r}+i c_{i}$. Знак $c_{i}$ представляет собой критерий устойчивости потока: отрищательное значение соответствует росту возмушений.

2.2. Акустические моды. Для двух акустических мод используется безвихревое поле скоростей:

$$
\frac{\partial u}{\partial z}-\frac{\partial w}{\partial x}=0
$$

В пределе $\mathrm{Re}^{-1}=0, R^{-1}=0, \phi=0$ уравнения (9)-(11) дают дисперсионное соотношение для акустических мод, которое напрямую связано с волновым оператором. Вектор, представляюший бегушую направо волну, имеет вид

$$
A_{1}=\left(\begin{array}{c}
p_{\mathrm{A} 1} \\
u_{A 1} \\
w_{A 1}
\end{array}\right)=\left(\begin{array}{c}
1 \\
-i \epsilon k \Delta^{-1 / 2} \\
\epsilon \frac{\partial}{\partial z} \Delta^{-1 / 2}
\end{array}\right) p_{\mathrm{A} 1} .
$$

Квадратный корень оператора $\Delta$ определяется как интегральньй оператор посредством преобразования Фурье. 
2.3. Проекторы в линейной задаче. Произвольное возмушение представляет собой сумму мод и, с учетом (14), (16), выглядит следующим образом:

$$
\left(\begin{array}{c}
p \\
u \\
w
\end{array}\right)=\left(\begin{array}{c}
p_{\mathrm{A} 1}+p_{\mathrm{A} 2}+p_{\mathrm{TS}} \\
H p_{\mathrm{A} 1}-H p_{\mathrm{A} 2}+K p_{\mathrm{TS}} \\
M p_{\mathrm{A} 1}-M p_{\mathrm{A} 2}+Q p_{\mathrm{TS}}
\end{array}\right),
$$

где операторы имеют вид

$$
\begin{gathered}
H=-\epsilon \Delta^{-1 / 2} i k, \quad M=\epsilon \Delta^{-1 / 2} \frac{\partial}{\partial z} \\
K=-\frac{1}{2 k^{2}} \frac{\partial}{\partial z} \frac{1}{\phi} \Delta, \quad Q=\frac{1}{2 \phi i k} \Delta .
\end{gathered}
$$

Связь (17) может рассматриваться как взаимно однозначное отображение динамических переменных, что немедленно дает проектор, выделяюший ТШ-волну:

$$
P_{\mathrm{TS}}=\left(\begin{array}{ccc}
0 & -2 k^{2} \Delta^{-1} \phi \frac{\partial}{\partial z} & -2 i k^{3} \Delta^{-1} \phi \\
0 & \frac{\partial^{2}}{\partial z^{2}} & i k \frac{\partial}{\partial z} \\
0 & i k \frac{\partial}{\partial z} & -k^{2}
\end{array}\right) \Delta^{-1} .
$$

Проектор для правой и левой акустических волн выписан в работе [6].

Эти проекторы обладают всеми свойствами ортогональных проекторов:

$$
P_{\mathrm{TS}}^{2}=P_{\mathrm{TS}}, \quad \ldots, \quad P_{\mathrm{TS}}+P_{\mathrm{A} 1}+P_{\mathrm{A} 2}=I, \quad P_{\mathrm{TS}} \cdot P_{\mathrm{A} 1}=\cdots=0 .
$$

Хотелось бы подчеркнуть неабелеву природу этих операторов.

Проекторы коммутируют с операторами $I \cdot(\partial / \partial t)$ и $L$; следовательно, действуя проекторами на уравнение (6), можно получить эволюционное уравнение соответствуюшей моды.

Действуя проектором $P_{\mathrm{TS}}$ на систему (6), получаем

$$
\begin{gathered}
\Delta \frac{\partial \Psi}{\partial t}+V_{0} \Delta \frac{\partial \Psi}{\partial x}-\frac{\partial \Psi}{\partial x} \frac{\partial \phi}{\partial z}-\mathrm{Re}^{-1} \Delta^{2} \Psi= \\
=\frac{\partial}{\partial z}\left[-u \frac{\partial u}{\partial x}-w \frac{\partial u}{\partial z}+\rho \frac{\partial p}{\partial x}\right]- \\
-\frac{\partial}{\partial x}\left[-u \frac{\partial w}{\partial x}-w \frac{\partial w}{\partial z}+\rho \frac{\partial p}{\partial z}\right] \\
u=\frac{\partial \Psi}{\partial z}+\epsilon \Delta^{-1 / 2} \frac{\partial p_{\mathrm{A} 1}}{\partial x}-\epsilon \Delta^{-1 / 2} \frac{\partial p_{\mathrm{A} 2}}{\partial x}, \\
w=-\frac{\partial \Psi}{\partial x}+\epsilon \Delta^{-1 / 2} \frac{\partial p_{\mathrm{A} 1}}{\partial z}-\epsilon \Delta^{-1 / 2} \frac{\partial p_{\mathrm{A} 2}}{\partial z}, \\
p=2 \frac{\partial^{2}}{\partial x^{2}} \Delta^{-1}(\phi \Psi)+p_{\mathrm{A} 1}+p_{\mathrm{A} 2} .
\end{gathered}
$$

Предел $\epsilon=0$ дает вихревую (ТШ) моду для несжимаемого потока $\rho_{\mathrm{TS}}=0$, поэтому

$$
\rho=\epsilon^{2} p_{\mathrm{A} 1}+\epsilon^{2} p_{\mathrm{A} 2} .
$$


Окончательно уравнение (20) принимает вид

$$
\begin{aligned}
\Delta \Psi_{t}+ & V_{0} \Delta \Psi_{x}-\Psi_{x} \phi_{z}-\operatorname{Re}^{-1} \Delta^{2} \Psi=-\Psi_{z} \Delta \Psi_{x}+\Psi_{x} \Delta \Psi_{z}- \\
& -\epsilon\left(\Delta \Psi \Delta^{1 / 2}\left(p_{\mathrm{A} 1}-p_{\mathrm{A} 2}\right)+\Delta \Psi_{x} \Delta^{-1 / 2}\left(p_{\mathrm{A} 1}-p_{\mathrm{A} 2}\right)_{x}+\right. \\
& \left.+\Delta \Psi_{z} \Delta^{-1 / 2}\left(p_{\mathrm{A} 1}-p_{\mathrm{A} 2}\right)_{z}\right)+O\left(\epsilon^{2}\right)
\end{aligned}
$$

Если $V_{0}=0, \phi=0$, простое самодействие дает известное уравнение для функции тока. Оно следует из формулы (23):

$$
\Delta \Psi_{t}-\operatorname{Re}^{-1} \Delta^{2} \Psi+\Psi_{z} \Delta \Psi_{x}-\Psi_{x} \Delta \Psi_{z}=0 .
$$

Действуя проектором $P_{\mathrm{A} 1}$ на систему (4), получаем эволюционное уравнение для данной моды, которое учитывает возмушения акустического давления:

$$
\begin{aligned}
\epsilon\left(\frac{\partial p_{\mathrm{A} 1}}{\partial t}+\right. & \left.V_{0} \frac{\partial p_{\mathrm{A} 1}}{\partial x}\right)+\Delta^{1 / 2} p_{\mathrm{A} 1}= \\
= & \frac{\epsilon}{2}\left[-\Psi_{z} p_{\mathrm{A} 1 x}+\Psi_{x} p_{\mathrm{A} 1 z}\right]+\epsilon \Delta^{-1 / 2}\left[-\Psi_{z} \Delta^{1 / 2} p_{\mathrm{A} 1 x}+\Psi_{x} \Delta^{1 / 2} p_{\mathrm{A} 1 z}\right. \\
& -2 \Psi_{x z} \Delta^{-1 / 2} p_{\mathrm{A} 1 x x}-2 \Psi_{z z} \Delta^{-1 / 2} p_{\mathrm{A} 1 x z}+2 \Psi_{x x} \Delta^{-1 / 2} p_{\mathrm{A} 1 x z}+ \\
& \left.\left.+2 \Psi_{x z} \Delta^{-1 / 2} p_{\mathrm{A} 1 z z}\right]+\Delta^{-1 / 2}\left[-\left(\Psi_{x z}\right)^{2}+\Psi_{x x} \Psi_{z z}\right)\right]+ \\
& +\epsilon\left[-\Psi_{z} \Delta^{-1}(\phi \Psi)_{x x x}+\Psi_{x} \Delta^{-1}(\phi \Psi)_{x x z}\right]+ \\
& +\epsilon \Delta^{-1} \phi \Delta^{-1} \frac{\partial^{2}}{\partial x^{2}}\left[\Psi_{z} \Delta \Psi_{x}-\Psi_{x} \Delta \Psi_{z}\right]+O\left(\epsilon^{2}\right) .
\end{aligned}
$$

В последнем выражении можно различить члены, которые связаны с взаимодействием (A1-TS) и генерацией (TS-TS). Эта система была применена для изучения сдвига кривой устойчивости акустическими методами в рамках теории возмушений операторов [7].

\section{3. ЛИНИИ ТОКА КАК КООРДИНАТЫ}

Поскольку функция тока определена, можно ввести линии тока как $\Psi=$ const $=n$. Линии тока и семейство ортогональных к ним кривых

$$
y=h(x, \tau)
$$

определяют новую криволинейную систему координат с переменными $(\tau, n)$. Эти переменные связаны с декартовыми координатами следующим образом:

$$
\tau=\varphi(x, y), \quad n=\Psi(x, y)
$$

Уравнение для функции $h(x, \tau)(25)$ легко может быть выведено из уравнения прямой, перпендикулярной линии тока в точке $X, Y$ :

$$
\frac{\partial \Psi}{\partial y}(X-x)-\frac{\partial \Psi}{\partial x}(Y-y)=0,
$$


которое дает

$$
\frac{d h}{d x}=\left[\frac{\partial \Psi / \partial y}{\partial \Psi / \partial x}\right]_{y=h}
$$

Это дифференциальное уравнение эквивалентно уравнению в полных дифференциалах вида

$$
\frac{\partial \Psi}{\partial x} d y-\frac{\partial \Psi}{\partial y} d x=0
$$

Форма Пфаффа в левой части последнего уравнения является точной тогда и только тогда, когда $\partial^{2} \Psi / \partial y^{2}+\partial^{2} \Psi / \partial x^{2}=0$, что означает $\operatorname{rot}_{z} v^{\prime}=0$. Такое условие сильно ограничивает выбор поля скоростей (сушествует потенциал поля скоростей, совпадающий с $\varphi$ ), отвечающего случаю отсутствия связанных с вязкостью членов.

В целом в двумерном случае установлено сушествование интегрируюшего множителя $\mu(x, y)$. Как уже упоминалось, мы отождествляем константу интегрирования для дифференциального уравнения (28) с переменной $\tau$ :

$$
\tau=\varphi(x, y)=-\int_{x_{0}}^{x} \mu\left(x^{\prime}, y_{0}\right) \Psi_{y}\left(x^{\prime}, y_{0}\right) d x^{\prime}+\int_{y_{0}}^{y} \mu\left(x, y^{\prime}\right) \Psi_{x}\left(x, y^{\prime}\right) d y^{\prime}
$$

Уравнение, связываюшее интегрируюший множитель $\mu$ и функцию $\Psi$, является прямым следствием условия интегрируемости:

$$
\frac{\partial \ln \mu}{\partial x} \frac{\partial \Psi}{\partial x}+\frac{\partial \ln \mu}{\partial y} \frac{\partial \Psi}{\partial y}+\Delta \Psi=0
$$

Назовем уравнение $\Delta \Psi+2 l \Psi_{x}+2 m \Psi_{y}+n \Psi=0$ "общим уравнением Лапласа". В таком случае уравнение (30) есть просто обшее уравнение Лапласа с $n=0$; можно проверить, что первый инвариант Лапласа $J=l_{y}-m_{x}$ обрашается в нуль. Следовательно, уравнение (30) заменой

$$
\Psi=\lambda \Omega
$$

$\lambda=1 / \sqrt{\mu}$, может быть преобразовано к виду (см., например, [8])

$$
\Delta \Omega+b \Omega=0, \quad b=-\frac{\Delta \ln \mu+\left(\ln _{x} \mu\right)^{2}+\left(\ln _{y} \mu\right)^{2}}{2} .
$$

Это есть уравнение Мутара [9], и сушествует связь с уравнением Лапласа

$$
\Delta \Phi=0
$$

Такая связь $\Omega=\widehat{M} \Phi$, где $\widehat{M}$ - преобразование Мутара, является достаточно обшей [9]; ее можно исследовать аналогично случаю уравнения Гурса [10]. Чтобы выявить главные этапы алгоритма, полезно следуюшее наблюдение:

$$
\left(\Delta+b^{\prime}\right) \Phi^{-1}=0
$$

если $b^{\prime}=-2\left(\Phi_{x}^{2}+\Phi_{y}^{2}\right) / \Phi^{2}$, и $\Delta \Phi=0$.

7 Теоретическая и математическая физика, т. 144, № 1, 2005 г. 
Приравнивая $b$ и $b^{\prime}$, получаем

$$
(\ln \Phi)_{x}^{2}+(\ln \Phi)_{y}^{2}=\frac{\Delta \ln \mu+\left(\ln _{x} \mu\right)^{2} / 2+\left(\ln _{y} \mu\right)^{2} / 2}{4}
$$

что дает класс интегрируюших множителей. Уравнение (35) обобшает условие, при котором второй инвариант Лапласа $K$ (пропорциональный $b$ ) обращается в нуль. Например, при $\mu=A(x) B(y), \Phi=s(x) p(y)$,

$$
b=\frac{\Delta \ln \mu+\left(\ln _{x} \mu\right)^{2} / 2+\left(\ln _{y} \mu\right)^{2} / 2}{2}=\frac{1}{2}\left(\ln _{x x} A+\ln _{y y} B+\ln _{x}^{2} A+\ln _{y}^{2} B\right),
$$

имеем для $A$ обыкновенное дифференциальное уравнение

$$
\ln _{x x} A+\frac{\left(\ln _{x} A\right)^{2}}{2}=4(\ln s)_{x}^{2}
$$

и аналогичное уравнение для $B$. Каждый конкретный выбор интегрируюшего множителя ведет к упрошению уравнения для $\Psi$ и дает частный случай системы $(23)$.

Для перехода в новую систему координат, такую как криволинейная система, нужно определить коэффициенты Ламе. Частные производные функций (26), условие ортогональности и определение функции тока определяют матрицу Ламе $\hat{h}$ :

$$
\hat{h}=\left(\begin{array}{ll}
\frac{\partial \Psi}{\partial x} & \frac{\partial \Psi}{\partial y} \\
\frac{\partial \varphi}{\partial x} & \frac{\partial \varphi}{\partial y}
\end{array}\right)=\left(\begin{array}{cc}
w & -u \\
\varphi_{x} & \frac{\varphi_{x} w}{u}
\end{array}\right), \quad \hat{h}^{-1}=\left(\begin{array}{cc}
\frac{\partial x}{\partial n} & \frac{\partial x}{\partial \tau} \\
\frac{\partial y}{\partial n} & \frac{\partial y}{\partial \tau}
\end{array}\right)=\frac{1}{\vec{v}^{2}}\left(\begin{array}{cc}
w & \frac{u^{2}}{\varphi_{x}} \\
-u & \frac{u w}{\varphi_{x}}
\end{array}\right)
$$

где для краткости мы заменили производные функции тока компонентами скорости: $\vec{v}^{2}=v^{2}+w^{2}$, а функции $x=x(n, \tau), y=y(n, \tau)$ определяют преобразование, обратное к (26). Следовательно, производные в выражениях (36) определены компонентами скорости и производной $\varphi_{x}$.

Окончательно компоненты метрического тензора $g_{i k}=g_{k i}$ криволинейной системы координат имеют следующий вид (в силу ортогональности отличны от нуля только диагональные компоненты):

$$
g_{n n}=\left(\frac{\partial x}{\partial n}\right)^{2}+\left(\frac{\partial y}{\partial n}\right)^{2}=\frac{1}{\vec{v}^{2}}
$$

и, аналогично, $g_{\tau \tau}=u^{2} /\left(\varphi_{x}^{2} \vec{v}^{2}\right)$. Эти компоненты и детерминант

$$
g=\left(\frac{u}{\varphi_{x} \vec{v}^{2}}\right)^{2}
$$

определяют дифференциальные операторы основных уравнений в векторном виде. Например, наиболее важный для дальнейшего лапласиан принимает вид

$$
\Delta \bar{v}=\left(\bar{\tau}-\frac{\bar{n} \varphi_{x}}{u}\right)\left|\bar{v}^{\prime}\right| \frac{\partial}{\partial n}\left(\frac{\left|\bar{v} \varphi_{x}\right|}{u} \frac{\partial\left(u / \varphi_{x}\right)}{\partial n}\right)
$$


где $\bar{\tau}, \bar{n}$ - единичные векторы. В терминах координат скорости уравнение (30) имеет вид $\left(\bar{v}^{\prime} \nabla\right) \ln \mu=-\operatorname{rot}_{z} \bar{v}^{\prime}$.

В случае $\mu=1(\Delta \Psi=0)$

$\varphi_{x}=-\Psi_{y}\left(x, y_{0}\right)+\int_{y_{0}}^{y} \Psi_{x x}\left(x, y^{\prime}\right) d y^{\prime}=-\Psi_{y}\left(x, y_{0}\right)-\int_{y_{0}}^{y} \Psi_{y y}\left(x, y^{\prime}\right) d y^{\prime}=-\Psi_{y}(x, y)=u$.

Мы разработали общую схему, однако сушествует много интересных конкретных способов выбора интегрируюшего множителя. Например, можно взять равным нулю второй инвариант Лапласа $(K=0)$, и в этом случае преобразование Лапласа (31) прямо приводит к уравнению Лапласа, которое легко интегрируется.

\section{4. РЕЗОНАНСНОЕ ВЗАИМОДЕЙСТВИЕ АКУСТИЧЕСКИХ МОД И МОДЫ ТШ}

Уравнения (23), (24) образуют связанную систему эволюционных уравнений для взаимодействуюших акустических и ТШ-мод. В случае генерации ТШ-моды в правых частях уравнений остается только акустический источник, т.е.

$$
\begin{aligned}
& \Delta \Psi_{t}+V_{0} \Delta \Psi_{x}-\Psi_{x} \cdot \phi_{z}-\operatorname{Re}^{-1} \Delta^{2} \Psi= \\
& =-\epsilon\left(\Delta \Psi \cdot \Delta^{1 / 2} p_{\mathrm{A} 1}+\Delta \Psi_{x} \cdot \Delta^{-1 / 2} p_{\mathrm{A} 1 x}+\Delta \Psi_{z} \cdot \Delta^{-1 / 2} p_{\mathrm{A} 1 z}\right), \\
& \epsilon\left(\frac{\partial p_{\mathrm{A}}}{\partial t}+V_{0} \frac{\partial p_{\mathrm{A}}}{\partial x}\right)+\Delta^{1 / 2} p_{\mathrm{A}}=\frac{\epsilon}{2}\left[-\Psi_{z} p_{\mathrm{A} x}+\Psi_{x} p_{\mathrm{A} z}\right]+ \\
& +\epsilon \Delta^{-1 / 2}\left[-\Psi_{z} \Delta^{1 / 2} p_{\mathrm{A} x}+\Psi_{x} \Delta^{1 / 2} p_{\mathrm{A} z}-2 \Psi_{x z} \Delta^{-1 / 2} p_{\mathrm{A} x x}-\right. \\
& \left.-2 \Psi_{z z} \Delta^{-1 / 2} p_{\mathrm{A} x z}+2 \Psi_{x x} \Delta^{-1 / 2} p_{\mathrm{A} x z}+2 \Psi_{x z} \Delta^{-1 / 2} p_{\mathrm{A} z z}\right] \text {. }
\end{aligned}
$$

Будем искать решение в виде

$$
\begin{aligned}
p_{\mathrm{A} 1}(x, z, t) & =A_{1}(\nu x, \nu t) \pi_{1} \exp \left(i\left(\omega_{1} t-k_{1} x\right)\right)+\cdots+\text { к.c. } \\
\Psi(x, z, t) & =B_{3}(\nu x, \nu t) \Psi_{3}(z) \exp \left(i\left(\omega_{3} t-k_{3} x\right)\right)+\cdots+\text { к.c. }
\end{aligned}
$$

Амплитуды $A_{1,2}, B_{3,4}$ являются медленно меняюшимися функциями переменных $x, t \mathrm{c}$ соответствующим малым параметром $\nu$. Профиль акустического поля поперек пограничного слоя $\pi_{1}$ удовлетворяет линейному уравнению

$$
i \omega_{1} \pi_{1}-i k_{1} V_{0} \pi_{1}+\epsilon^{-1} \Delta^{1 / 2} \pi_{1}=0 .
$$

Разумный с точки зрения соотношения длины звуковой волны и ширины пограничного слоя $l_{0}$ выбор параметров дает приближенное уравнение для профиля звуковой волны со спектральным параметром $k_{1}$ :

$$
\left(1-\frac{k_{1}}{\omega_{1}} V_{0}\right) \pi_{1}+\frac{k_{1}}{\omega_{1} \epsilon}\left(1-\frac{1}{2 k_{1}^{2}} \partial_{z}^{2}\right) \pi_{1}=0 .
$$


Собственные функции нормированы следующим образом: $\int_{0}^{\delta} V_{0} \pi_{1}^{2}(z) d z=1$. Рассмотрим условие четырехволнового резонанса. Исследование алгебраических соотношений между параметрами дает соответствующие условия

$$
\omega_{2}=\omega_{1}+\omega_{3}, \quad \omega_{4}=\omega_{3}-\omega_{1}
$$

Акустические волновые числа являются вещественными, волновые числа ТШ-моды могут быть комплексными. Подставляя формулы (42), (43) в формулы (41), (40) и оставляя только резонансные члены, мы приходим к подсистеме, которая затем проектируется на поперечные моды, выделенные из уравнения ОЗ, как на базис.

Другой пример возникает из акустической задачи (не ортогональной). Положим $\nu=1$ :

$$
\begin{aligned}
A_{1 t}+c_{\mathrm{A} 1} A_{1 x} & =n_{\mathrm{A} 1} A_{2} B_{3}^{*} e^{i(\Delta k) x}, \\
A_{2 t}+c_{\mathrm{A} 2} A_{2 x} & =n_{\mathrm{A} 2} A_{1} B_{3}^{*} e^{i(-\Delta k) x}, \\
B_{3 t}+c_{\mathrm{TS} 1} B_{3 x} & =-\epsilon n_{\mathrm{TS} 1} A_{1} B_{4} e^{i\left(\Delta k^{\prime}\right) x}, \\
B_{4 t}+c_{\mathrm{TS} 2} B_{4 x} & =-\epsilon n_{\mathrm{TS} 1} A_{1}^{*} B_{3} e^{i\left(-\Delta k^{\prime}\right) x} .
\end{aligned}
$$

Групповые скорости и нелинейные константы выражаются через интегралы по ширине пограничного слоя $\delta$ :

$$
\begin{aligned}
& c_{\mathrm{A} 1}=\int_{0}^{\delta}\left[\pi_{1}^{2}-\epsilon^{-1} i k_{1} \pi_{1} \int_{0}^{z^{\prime}} \pi_{1} d z\right] d z^{\prime} \\
& n_{\mathrm{A} 1}=\int_{0}^{\delta}\left[1.5\left(-i k_{3}^{*} \Psi_{3}^{*} \pi_{2 z}+i k_{2} \Psi_{3 z}^{*} \pi_{2} t\right)+\right. \\
& \left.+\int_{0}^{z^{\prime}} \pi_{1}\left(-i k_{3}^{*} \Psi_{3 z}^{*} \pi_{2 z}+i k_{2} \Psi_{3 z z}^{*} \pi_{2}\right) d z\right] d z^{\prime}, \\
& c_{\mathrm{A} 2}=\int_{0}^{\delta}\left[\pi_{2}^{2}-\epsilon^{-1} i k_{2} \pi_{2} \int_{0}^{\delta} \pi_{2} d z\right] d z^{\prime} \text {, } \\
& n_{\mathrm{A} 2}=\int_{0}^{\delta} \pi_{2}\left[\left(1.5\left(-i k_{3} \Psi_{3} \pi_{1 z}+i k_{1} \Psi_{3 z} \pi_{1}\right)+\right.\right. \\
& \left.\left.+\int_{0}^{z^{\prime}}\left(-i k_{3} \Psi_{3 z} \pi_{1 z}+i k_{1} \Psi_{3 z z} \pi_{1}\right) d z\right)\right] d z^{\prime}, \\
& c_{\mathrm{TS} 1}=\frac{\int_{0}^{\delta} \Psi_{3}\left[\left(V_{0}+i 4 k_{3} R e^{-1}\right) \Psi_{3 z z}+\left(2 k_{3} \omega_{3}+\varphi_{z}\right) \Psi_{3}\right] d z}{\int_{0}^{\delta} \Psi_{3} \Psi_{3 z z} d z}, \\
& n_{\mathrm{TS} 1}=\frac{\int_{0}^{\delta} \Psi_{3}\left(\Psi_{4 z z} \pi_{1 z}-k_{1} k_{4} \Psi_{4 z z} \int_{0}^{z^{\prime}} \pi_{1} d z+\Psi_{4 z z z} \pi_{1}\right) d z^{\prime}}{\int_{0}^{\delta} \Psi_{3} \Psi_{3 z z} d z} \\
& c_{\mathrm{TS} 2}=\frac{\int_{0}^{\delta} \Psi_{4}\left[\left(V_{0}+i 4 k_{4} R e^{-1}\right) \Psi_{4 z z}+\left(2 k_{4} \omega_{4}+\varphi_{z}\right) \Psi_{4}\right] d z}{\int_{0}^{\delta} \Psi_{4} \Psi_{4 z z} d z}, \\
& n_{\mathrm{TS} 2}=\frac{\int_{0}^{\delta} \Psi_{4}\left(\Psi_{3 z z} \pi_{1 z}^{*}-k_{1} k_{3} \Psi_{3 z z} \int_{0}^{z^{\prime}} \pi_{1}^{*} d z+\Psi_{3 z z z} \pi_{1}^{*}\right) d z^{\prime}}{\int_{0}^{\delta} \Psi_{4} \Psi_{4 z z} d z} .
\end{aligned}
$$


Структура полученных уравнений есть частный случай структуры для общей $N$-волновой системы (об интегрировании таких систем см. [11]).

\section{5. ЗАКЛЮЧЕНИЕ}

В настоящей работе на основе объединенного преобразования Лапласа-Мутара решена задача о нахождении интегрирующего множителя для построения ортогональных криволинейных координат для линий $(2+1)$-мерного вязкого потока. Функция тока представляет собой вихревую моду с отвечающим ей проектором, которому сопутствуют два ортогональных проектора для акустических возмушений в пограничном слое. Основная нелинейная система переписывается в переменных, соответствуюших этим модам. Полученная в результате система $(20),(24)$ и уравнение для распространяющихся в противоположных направлениях акустических мод могут рассматриваться как основная система для описания всех возмушений в пограничном слое. Принимаемая обычно медленная зависимость потока в пограничном слое от переменной $x$ не меняет общей структуры выражений. Как резонансные, так и нерезонансные процессы могут изучаться отдельно от акустических волн. Эти моды имеют различный масштаб, следовательно, численное и аналитическое моделирование и управление должны оказаться более эффективными.

Благодарности. Авторы благодарны В. Левандовскому за обсуждения. Работа поддержана грантом Польского министерства научных исследований и информационных технологий PBZ-Min-008/P03/2003.

\section{Список литературы}

[1] S. B. Leble. Nonlinear Waves in Waveguides. Berlin: Springer, 1991.

[2] B.-T. Chu, L. S. G. Kovasznay. J. Fluid Mech. 1958. V. 3. P. 494

[3] X. Wu. J. Fluid Mech. 2001. V. 431. P. 91.

[4] А. И. Рубан. Изв. АН СССР. Мех. жидк. и газа. 1984. № 5. С. 44; Fluid Dyn. 1985. V. 19. P. 709 .

[5] A. A. Perelomova. Appl. Math. Lett. 2000. V. 13. P. 93; Acta Acustica. 1998. V. 84. P. 1002.

[6] S. Leble, A. Perelomova. Tollmienn-Schlichting and sound waves interaction: nonlinear resonances. In: Nonlinear acoustics at the beginning of the 21th century (16th Int. Symp. on Nonlinear Acoustics). Eds. O. Rudenko, O. Sapozhnikov. M.: MGU, 2002. P. 203.

[7] С. В. Лебле, И. Ю. Попов, Ю. В. Гугель. Гидромеханика. 1993. № 67. С. 3.

[8] Е. И. Ганжа. Каскадный метод Лапласа. Красноярск: Изд-во Красноярского педагогического ун-та, 2000.

[9] C. Athorne, J. J. C. Nimmo. Inverse Problems. 1991. V. 7. P. 809

[10] S. Leble, A. Yurov. J. Math. Phys. 2002. V. 43. P. 1095.

[11] С. Лебле. ТМФ. 2000. Т. 122. № 2. С. 239. 